\title{
Nephron-sparing approaches in the management of upper tract urothelial carcinoma: indications and clinical outcomes
}

\author{
Young Hwii Ko \\ Department of Urology, College of Medicine, Yeungnam University, Daegu, Republic of Korea \\ Correspondence to: Young Hwii Ko. Department of Urology, College of Medicine, Yeungnam University, 317-1 Daemyung-dong, Nam-gu, Daegu \\ 705-035, Republic of Korea. Email: urokyh@naver.com.
}

\begin{abstract}
Given the more aggressive phenotype and prognosis of the upper tract urothelial carcinoma (UTUC) than those of bladder tumor, radical removal of the entire ureter along with ipsilateral kidney has long been the standard of care. However, recent advances in diagnostic imaging and endoscopic armamentarium have markedly enhanced the role of nephron-sparing approaches for well-selected cases, especially with low-risk features. Historically, this strategy was exclusively considered for managing a patient unfit to undergo radical nephroureterectomy (RNU). After observing effective oncologic control for these imperative cases, an elective risk-based indication was introduced with care. Two representative modalities in this strategy include endoscopic management via retrograde ureterorenoscopy (URS) or antegrade percutaneous approach and segmental ureterectomy (SU). SU, which includes different types of unstandardized procedures, is the most widely reported alternative to RNU. Despite the lack of qualified evidence regardless of the strategy applied, systemic reviews have consistently revealed similar survival after endoscopic management and RNU for low-grade and noninvasive UTUC. However, selected patients with high-grade and invasive UTUC could benefit from SU while maintaining the oncologic outcomes observed after RNU. Based on these findings, the currently available guidelines have extended the use of the nephronsparing approach gradually, recommending segmental resection especially for the distal ureteral tumors even in patients with high-risk features. Nonetheless, the fact that evidence supporting these conclusions remains poor and potentially biased cannot be overemphasized.
\end{abstract}

Keywords: Nephron-sparing surgery; segmental ureterectomy (SU); upper tract urothelial carcinoma (UTUC)

Submitted Dec 29, 2019. Accepted for publication Mar 13, 2020.

doi: $10.21037 /$ tcr.2020.03.66

View this article at: http://dx.doi.org/10.21037/tcr.2020.03.66

\section{Introduction}

Radical nephroureterectomy (RNU) has been recommended as the standard of care in the management of upper tract urothelial carcinoma (UTUC) for a long time (1). However, despite complete removal of the entire ipsilateral ureter with a functional kidney, the recurrence of the tumor cannot be prevented effectively. Moreover, it is associated with formidable morbidity induced by reduced renal function. Particularly, intravesical recurrence of tumor, which has been reported to occur after $22-47 \%$ of the cases of RNU, remains a clinical dilemma $(2,3)$. The loss of ipsilateral kidney does not routinely require prolonged hemodialysis except in the elderly or case of renal insufficiency. However, decreased glomerular filtration rate, which is frequently encountered even in relatively young and healthy patients postoperatively, potentially diminishes the oncologic controllability by reducing the effective dosage of the adjuvant chemotherapy. Additionally, there has been a debate over the clinical relevance of radical removal of an affected organ regardless of the volume and the nature of the tumor within the ureter $(4,5)$. With this background, several nephron-sparing strategies replacing RNU have been suggested, which are gaining enthusiasm as alternative conservative surgical management strategies for UTUC. Currently, segmental ureterectomy (SU) with 
or without the psoas hitch or the Boari flap and endoscopybased procedures including ureterorenoscopy (URS) or percutaneous approach for the low-risk disease are the most widely reported nephron-sparing strategies.

Traditionally, a nephron-sparing approach was applied to a small number of cases under certain circumstances where RNU was hard to perform safely. The imperative indications for conservative management of UTUC include bilateral disease, solitary functioning kidney, chronic kidney disease/renal insufficiency, and hereditary predisposition (6). Based on the accumulation of clinical experiences that help in selecting proper patients with conditions other than the aforementioned ones, current indications for the conservative approaches were expanded. However, to accept these approaches as elective procedures in the clinical practice, the following key questions should be answered: (I) is the tumor recurrence within the remaining ureter acceptable? (II) Does not the procedure provoke intravesical tumor recurrence? (III) What is the proper surveillance strategy after each procedure? Focused on endoscopy-based approaches and SU, we aimed to review the indications and the current clinical outcomes.

\section{General indications for nephron-sparing surgery}

Due to the more aggressive disease phenotype of UTUC than of bladder tumor, radical removal of the affected ureter in UTUC was suggested, especially for high-risk disease. In contrast, despite the lack of qualified evidence, conservative strategies have been used for patients with relatively low-risk characteristics. Due to its narrow diameter and inner anatomic location, accurate clinical staging of UTUC is challenging. Ureteroscopy inspection with/without biopsy has become the most widely used modality in the identification and localization of UTUC. However, the alleged risk of intravesical tumor recurrence after the procedure remains a topic of clinical debate. Despite these limitations, risk stratification based on several clinicopathologic characteristics has been suggested to guide the decision-making.

The European Association of Urology (EAU) guidelines stratify UTUC patients into low- or high-risk patients based on clinical, endoscopic, radiographic, and histopathologic factors (6). Low-risk cases include unifocal disease, tumor size $<2 \mathrm{~cm}$, low-grade cytology and/or URS biopsy, and noninvasive disease on imaging. High-risk cases include hydronephrosis, multifocal disease, tumor size $\geq 2 \mathrm{~cm}$, high- grade cytology and/or URS biopsy, invasive disease on imaging, and prior radical cystectomy.

National Comprehensive Cancer Network (NCCN) guidelines stratify UTUC into cases having favorable or less favorable clinical and pathological criteria for nephron preservation (7). Favorable cases include low-grade tumors based on cytology and biopsy, papillary architecture, tumor size $<1.5 \mathrm{~cm}$, unifocal tumor, and cross-sectional imaging showing no concern for invasive disease. Less favorable cases include multifocal tumors, flat or sessile tumor architecture, tumor size $\geq 1.5 \mathrm{~cm}$, high-grade tumors, cT2T4 tumors, mid-ureteral and proximal ureteral tumors (due to technical challenges), and tumors crossing infundibulum or the ureteropelvic junction.

\section{Endoscopic management of UTUC}

Retrograde URS or antegrade percutaneous approaches were attempted in a small subgroup of patients with imperative indication. However, with increased experience, they were also attempted in elective patients with a lowrisk profile. Tumors present in up to the middle and distal ureter could generally be accessed using semi-rigid URS. However, with newer advances and widespread use of flexible devices, retrograde endoscopic ablation has gained popularity. To obtain maximal debulking of the tumor within the ureter, the usage of flexible URS rather than a rigid one is recommended (6).

\section{The technique of retrograde ureteroscopic or anterograde percutaneous tumor ablation}

Endoscopic ablation techniques have generally described an initial debulking with cold cup or basket followed by treatment with electrocautery or laser ablation (7-10). However, among several methods of ablation, the use of laser for treatment is recommended until complete tumor resection or destruction can be achieved (6,7). Interest in electrocautery has waned due to transmural injury to the urothelium, which contributes to postoperative strictures. Complications associated with ureteroscopic ablation remain low (8-13\% of cases) (11). The risk of ureteral or renal perforation is $2-4 \%$, while stricture rates are $5-14 \%$ (12-14).

For the tumors located in the lower caliceal system that are inaccessible or difficult to manage even with flexible ureteroscopy, percutaneous access can be utilized. The 
principal advantage of the percutaneous approach is the ability to use larger diameters of endoscopic instruments for more efficient resection (11). Apart from the instruments for URS, resectoscope with loop cautery, a procedure similar to transurethral resections for the bladder tumors, can be used to effectively debulk the tumors (15). The primary disadvantages of the percutaneous approach include greater invasiveness compared to the retrograde approach, violation of the closed urinary system, and thereby the risk of tumor seeding of the nephrostomy tube tract $(16,17)$. The complication rate of the percutaneous approach is higher than URS (11). The reported overall complication rate was $27 \%$ including $17 \%$ risk of transfusion, $2 \%$ risk of renal failure, and $1 \%$ risk of emergency nephrectomy or angioembolization (18).

\section{Oncologic outcomes}

A vast majority of reported literature regarding the endoscopy-based approach includes small retrospective series without a description of clear selection criteria for this procedure. There are no reported randomized studies and the data encompass fewer than 100 patients. Additionally, many series had predominantly low-grade tumors with small sizes. Systemic reviews of retrospective studies on this issue, however, generated consistent conclusions from heterogeneous evidence $(11,18,19)$. Recurrence of UTUC is common and occurs in most of the patients, which mandates regular surveillance of the disease (18). Thus, the endoscopy-based approach is indicated for compliant patients who will adhere to a strict follow-up regimen (11). For elective cases with selected favorable disease characteristics, endoscopic management appears to provide effective oncological control (disease-specific survival of 5 years) and renal preservation. However, this has to be balanced against the risk of tumor progression and lower relapse-free survival than RNU outcomes $(18,20)$. Among the variables associated with tumor recurrence, several reports have consistently demonstrated a strong association between recurrence and tumor grade $(11,18)$. In the largest retrospective series with 141 patients with a median followup of 66 months who underwent a percutaneous approach over 30 years, ipsilateral tumor recurrence was observed in $37 \%$ of the patients with the low-grade disease and in $63 \%$ of the patients with high-grade disease. In this series, the grade was the only predictor of tumor recurrence (hazard ratio: $2.12, \mathrm{P}=0.018)(21)$. Although there are no reported randomized controlled trials, systemic reviews have shown that nephron-sparing strategies show similar survival outcomes when compared with RNU for low-risk patients or patients with favorable disease criteria (19-22). In contrast, they also demonstrated that there is no place for endoscopic management for unfavorable disease in elective patients.

During counseling with patients, the risks of poor oncologic control and tumor progression with endoscopic management must be weighed against the perioperative risks such as poor life expectancy associated with end-stage renal failure and consequent hemodialysis involved with major surgery such as RNU (18).

\section{Tumor recurrence within the urinary tract including the ipsilateral ureter}

Recurrence rates following endoscopic management for UTUC are fairly high. The upper tract recurrence in retrograde approaches ranged from $15 \%$ to $90 \%$ (11). However, disease progression to RNU occurred in 3-33\% of the cases and cancer-specific survival (CSS) remained high $(70-100 \%)(11)$, which makes this approach an acceptable option for selected cases.

Several attempts to reduce the probability of tumor recurrence in ipsilateral ureter have been suggested including instillation of bacillus Calmette-Guerin (BCG) vaccine or mitomycin $\mathrm{C}$. Especially for patients with carcinoma in situ, BCG immunotherapy appears to have the best response. However, further investigations on optimizing the drug delivery to the upper tract are needed. The feasibility of antegrade instillation via the three-valve system has been proven $(23,24)$. Retrograde instillation through a ureteric stent can be an alternative way, but it can be dangerous due to possible ureteric obstruction and consecutive pyelovenous influx during instillation (6).

Two prospective randomized trials reported that adjuvant instillation of intravesical agents decreased the recurrence following RNU $(25,26)$. However, this approach needs to be studied in an endoscopic UTUC ablation series. In a few series that reported intravesical recurrence, the intravesical recurrence-free survival was similar for the endoscopic management and the RNU groups (19).

\section{Proper surveillance strategy after endoscopic tumor ablation procedure}

Long-term surveillance of over 5 years including urine cytology, radiologic evaluation of the upper tract, and 
endoscopic inspection are required following nephronsparing treatment due to a high-risk of disease recurrence (7). Stage pT0 or pT1 tumors should be followed-up with serial cystoscopies at 3-month intervals for the first year and longer intervals in case of a negative test. Nevertheless, the surgeon keeps in mind that endoscopic management comes with a risk of understating and undergrading (6). Thus, these tumors should be followed-up with ureteroscopy and upper tract imaging at 3-to 12 -month intervals if endoscopic resection is considered $(6,7)$.

Due to the limitations in detection and identification of UTUC using currently available radiologic evaluation techniques, the need for novel imaging technology is growing. Narrow-band imaging and photodynamic techniques plausibly help in early detection of the tumor during follow-up. Confocal laser endomicroscopy, which uses fiber-optic imaging probes for in vivo highresolution visualization of cellular architecture and morphology, presents another potential tool to detect viable carcinoma (27).

\section{SU}

Over the past decades, $\mathrm{SU}$ has been increasingly reported at high-volume centers with favorable perioperative, functional, and oncologic outcomes (28-33). SU with wide margins provides adequate pathologic specimens for staging and grading while completely preserving ipsilateral renal function. Despite heterogeneous patient and tumor characteristics, several retrospective series have consistently demonstrated similar oncologic outcomes after SU and standard RNU in terms of overall survival, CSS, and intravesical tumor recurrences $(6,19,34)$. However, readers should keep in mind that the inclusion criteria and surgical tasks for the SU techniques are not standardized yet. There is substantial diversity in the techniques of performing $\mathrm{SU}$, dominantly dependent on the level and the extent of the tumor within the affected ureter. The most "radical" and extirpative way of performing SU would be a complete ureterectomy with ileal ureter replacement. However, the most frequently reported way is distal ureterectomy, in which the most radical approach involves distal ureterectomy with bladder cuffing for distal ureteral tumors with concomitant ureteroneocystostomy and ipsilateral pelvic nodal dissection. However, in many reports, the degree and the implementation of bladder cuffing and lymphadenectomy have not been clearly described. In contrast, reports on ureteroureterostomy, the simplest way to do perform SU for proximal and mid-ureteral tumors, are relatively sparse.

\section{Technique of SU}

Despite the lack of standardization, the fundamental principles for SU include (I) atraumatic, "no-touch" ureteral dissection, (II) identification of the limits of the ureteral tumor (with or without the use of concomitant ureteroscopy), (III) isolation of the affected ureteral segment to prevent tumor spillage (35), and (IV) tumor resection with adequate $(1-2 \mathrm{~cm})$ safety margins (36). We also recommend preparing a frozen section to verify the absence of the remaining tumor within the ipsilateral ureter.

\section{Oncologic outcomes}

While the number of reports and cases of SU transcend those of endoscopic management for UTUC, the majority of them were from retrospective small series. Several systemic reviews of retrospective trials demonstrated acceptable oncologic safety. These results suggested that SU should be used as the first line of treatment for lowrisk UTUC. When compared with standard RNU, no significant differences were found in the 3-, 5-year, or the last follow-up CSS (19). Interestingly, these results were consistent in stage and grade-based subgroup analysis. Additionally, no significant differences were found between SU and RNU in univariate and multivariate analyses in terms of CSS or any other oncologic outcomes, despite an overall trend toward worse outcomes after SU $(19,34)$.

EAU guidelines have recommended SU for low-risk disease. For mid-ureteral or proximal ureteral lesion, ureteroureterostomy could be an alternative option. For high-risk cancer with an imperative condition, distal ureterectomy with/without lymph node dissection could be an alternative option, but the grade of recommendation is not high (grade C). In the case of a distally located tumor, complete distal ureterectomy with neocystostomy with/without lymph node dissection could be selectively recommended in high-risk patients (6).

The NCCN guidelines selectively recommend distal ureterectomy with ureteral reimplantation and regional lymphadenectomy (in high-grade tumors) only for distal ureteral tumors (7). For upper or mid-ureteral tumors, no guidelines recommend $\mathrm{SU}$ as the primary treatment option. 


\section{Tumor recurrence within the urinary tract including the ipsilateral ureter}

The reported upper tract recurrence rate is between $4.1 \%$ and $7 \%$ with a mean time to the procedure of $33.3-$ 54 months $(37,38)$. A recent propensity-matched Korean series from a high-volume center reported a recurrence rate of $6.8 \%$ for distal ureterectomy and bladder cuffing (39). Therefore, prolonged surveillance of the ipsilateral ureter via computed tomography (CT) or ureteroscopy is essential. Similar to the findings regarding local recurrence, the incidence of metastatic recurrence and bladder recurrence in SU was similar to that in RNU (19).

\section{Proper surveillance strategy after endoscopic tumor ablation procedure}

For patients treated with nephron-sparing treatment, follow-up should be more frequent and stricter than that for patients treated with RNU. Urine cytology and CT should be performed at 3 and 6 months after nephron-sparing treatment. Subsequent cytological and CT investigations should be performed annually according to EAU guidelines. To evaluate the remaining urinary tract, cystoscopy, ureteroscopy, and cytology are recommended at 3 months, at 6 months, at every 6 months after that for 2 years and annual investigations thereafter (6).

According to the NCCN guidelines, imaging of the upper tract collecting system or ureteroscopy at 3- to 12 -month intervals is recommended for patients treated with nephron-sparing surgery. Radiologic evaluation includes abdominal/pelvic CT, magnetic resonance imaging with or without contrast, and chest imaging (7).

\section{Summary}

Despite growing interest in the nephron-sparing strategy for UTUC, the level of evidence from contemporary studies is not high enough, implying that this approach is not suitable for every patient with UTUC. Among two representative way including endoscopic management and $\mathrm{SU}$, endoscopic management was exclusively considered for managing a patient unfit to undergo RNU. SU, which includes different types of unstandardized procedures, is the most widely reported alternative to RNU. Pieces of literature have consistently demonstrated that selected patients with high-grade and invasive UTUC could benefit from SU while maintaining the oncologic outcomes observed after RNU. Nevertheless, elective patients should undergo preoperative counseling for the increased risk of local recurrence, which has been reported in up to half of the cases. Stringent surveillance is required for patients during the follow-up period. Thus, the benefit of preserving renal function and the increased risk of tumor recurrence should be balanced by careful counseling.

\section{Acknowledgments}

Funding: None.

\section{Footnote}

Provenance and Peer Review: This article was commissioned by the Guest Editor (Ja Hyeon Ku, Hyeong Dong Yuk, Hyung Suk Kim) for the series "Urothelial Carcinoma" published in Translational Cancer Research. The article was sent for external peer review organized by the Guest Editor and the editorial office.

Conflicts of Interest: The author has completed the ICMJE uniform disclosure form (available at http://dx.doi. org/10.21037/tcr.2020.03.66). The series "Urothelial Carcinoma" was commissioned by the editorial office without any funding or sponsorship. The author has no other conflicts of interest to declare.

Ethical Statement: The author is accountable for all aspects of the work in ensuring that questions related to the accuracy or integrity of any part of the work are appropriately investigated and resolved.

Open Access Statement: This is an Open Access article distributed in accordance with the Creative Commons Attribution-NonCommercial-NoDerivs 4.0 International License (CC BY-NC-ND 4.0), which permits the noncommercial replication and distribution of the article with the strict proviso that no changes or edits are made and the original work is properly cited (including links to both the formal publication through the relevant DOI and the license). See: https://creativecommons.org/licenses/by-nc-nd/4.0/.

\section{References}

1. Redrow GP, Matin SF. Upper tract urothelial carcinoma: epidemiology, high risk populations and detection.

Minerva Urol Nefrol 2016;68:350-58. 
2. Wu WJ, Ke HL, Yang YH, et al. Should patients with primary upper urinary tract cancer receive prophylactic intravesical chemotherapy after nephroureterectomy? J Urol 2010;183:56-61.

3. Xylinas E, Colin P, Audenet F, et al. Intravesical recurrence after radical nephroureterectomy for upper tract urothelial carcinomas: predictors and impact on subsequent oncological outcomes from a national multicenter study. World J Urol 2013;31:61-8.

4. Seisen T, Granger B, Colin P, et al. A systematic review and meta-analysis of clinicopathologic factors linked to intravesical recurrence after radical nephroureterectomy to treat upper tract urothelial carcinoma. Eur Urol 2015;67:1122-33.

5. Miyake H, Hara I, Kamidono S, et al. Multifocal transitional cell carcinoma of the bladder and upper urinary tract: molecular screening of clonal origin by characterizing CD44 alternative splicing patterns. J Urol 2004;172:1127-9.

6. Rouprêt M, Babjuk M, Compérat E, et al. European Association of Urology guidelines on upper urinary tract urothelial carcinoma: 2017 update. Eur Urol 2018;73:111-22.

7. National Comprehensive Cancer Network. National Comprehensive Cancer Network clinical practice guidelines in oncology, bladder cancer, version 1. 2020. Available online: https://www.nccn.org/professionals/ physician_gls/default.aspx

8. Fiuk JV, Schwartz BF. Upper tract urothelial carcinoma: paradigm shift towards nephron sparing management. World J Nephrol 2016;5:158-65.

9. Motamedinia P, Hoenig D, Okeke Z, et al. A case for nephron sparing surgery in the management of upper tract urothelial carcinoma. J Endourol 2016;30:S18-22.

10. Suriano F, Brancato T. Nephron-sparing management of upper tract urothelial carcinoma. Rev Urol 2014;16:21-8.

11. Raman JD, Park R. Endoscopic management of uppertract urothelial carcinoma. Expert Rev Anticancer Ther 2017;17:545-54.

12. Raman JD. Kidney sparing surgery for uppertract urothelial carcinoma. Minerva Urol Nefrol 2016;68:359-71.

13. Seisen T, Colin P, Rouprêt M. Risk-adapted strategy for the kidney-sparing management of upper tract tumours. Nat Rev Urol 2015;12:155-66.

14. Soderdahl DW, Fabrizio MD, Rahman NU, et al. Endoscopic treatment of upper tract transitional cell carcinoma. Urol Oncol 2005;23:114-22.
15. Jabbour ME, Desgrandchamps F, Cazin S, et al. Percutaneous management of grade II upper urinary tract transitional cell carcinoma: the long-term outcome. J Urol 2000;163:1105-7; quiz 1295.

16. Jabbour ME, Smith AD. Primary percutaneous approach to upper urinary tract transitional cell carcinoma. Urol Clin North Am 2000;27:739-50.

17. Liatsikos EN, Dinlenc CZ, Kapoor R, et al. Transitionalcell carcinoma of the renal pelvis: ureteroscopic and percutaneous approach. J Endourol 2001;15:377-83; discussion 397.

18. Cutress ML, Stewart GD, Zakikhani P, et al. Ureteroscopic and percutaneous management of upper tract urothelial carcinoma (UTUC): systematic review. BJU Int 2012;110:614-28.

19. Seisen T, Peyronnet B, Dominguez-Escrig JL, et al. Oncologic outcomes of kidney-sparing surgery versus radical nephroureterectomy for upper tract urothelial carcinoma: a systematic review by the EAU non-muscle invasive bladder cancer guidelines panel. Eur Urol 2016;70:1052-68.

20. Margulis V, Shariat SF, Matin SF et al. Outcomes of radical nephroureterectomy: a series from the Upper Tract Urothelial Carcinoma Collaboration. Cancer 2009;115:1224-33.

21. Motamedinia P, Keheila M, Leavitt DA, et al. The expanded use of percutaneous resection for upper tract urothelial carcinoma: a 30-year comprehensive experience. J Endourol 2016;30:262-7.

22. Yakoubi R, Colin P, Seisen T, et al. Radical nephroureterectomy versus endoscopic procedures for the treatment of localised upper tract urothelial carcinoma: a meta-analysis and a systematic review of current evidence from comparative studies. Eur J Surg Oncol 2014;40:1629-34.

23. Redrow GP, Guo CC, Brausi MA, et al. Upper urinary tract carcinoma in situ: current knowledge, future direction. J Urol 2017;197:287-95.

24. Giannarini G, Kessler TM, Birkhauser FD, et al. Antegrade perfusion with bacillus Calmette-Guerin in patients with non-muscle-invasive urothelial carcinoma of the upper urinary tract: who may benefit? Eur Urol 2011;60:955-60.

25. O'Brien T, Ray E, Singh R, et al. Prevention of bladder tumours after nephroureterectomy for primary upper urinary tract urothelial carcinoma: a prospective, multicentre, randomised clinical trial of a single postoperative intravesical dose of mitomycin $\mathrm{C}$ (the 
ODMIT-C Trial). Eur Urol 2011;60:703-10.

26. Miyamoto K, Ito A, Wakabayashi M, et al. A Phase III trial of a single early intravesical instillation of pirarubicin to prevent bladder recurrence after radical nephroureterectomy for upper tract urothelial carcinoma (JCOG1403, UTUC THP Phase III). Jpn J Clin Oncol 2018;48:94-7.

27. Chen SP, Liao JC. Confocal laser endomicroscopy of bladder and upper tract urothelial carcinoma: a new era of optical diagnosis? Curr Urol Rep 2014;15:437.

28. Rouprêt M, Harmon JD, Sanderson KM, et al. Laparoscopic distal ureterectomy and anastomosis for management of lowrisk upper urinary tract transitional cell carcinoma: preliminary results. BJU Int 2007;99:623-7.

29. McClain PD, Mufarrij PW, Hemal AK. Robot-assisted reconstructive surgery for ureteral malignancy: analysis of efcacy and oncologic outcomes. J Endourol 2012;26:1614-7.

30. Krane LS, Hemal AK. Surgeon-controlled robotic ureteral surgery. Curr Opin Urol 2012;22:70-7.

31. Hemal AK, Stansel I, Babbar P, et al. Robotic-assisted nephroureterectomy and bladder cuff excision without intraoperative repositioning. Urology 2011;78:357-64.

32. Zargar H, Krishnan J, Autorino RM, et al. Robotic nephroureterectomy: a simplifed approach requiring no patient repositioning or robot redocking. Eur Urol 2014;66:769-77.

33. Pathak RA, Hemal AK. Techniques and outcomes

Cite this article as: Ko YH. Nephron-sparing approaches in the management of upper tract urothelial carcinoma: indications and clinical outcomes. Transl Cancer Res 2020;9(10):6589-6595. doi: $10.21037 /$ tcr.2020.03.66 of robotassisted nephroureterectomy for upper tract urothelial carcinoma. Eur Urol Focus 2018;4:657-61.

34. Fang D, Seisen T, Yang K, et al. A systematic review and meta-analysis of oncological and renal function outcomes obtained after segmental ureterectomy versus radical nephroureterectomy for upper tract urothelial carcinoma. Eur J Surg Oncol 2016;42:1625-35.

35. Glinianski M, Guru KA, Zimmerman G, et al. Robotassisted ureterectomy and ureteral reconstruction for urothelial carcinoma. J Endourol 2009;23:97-100.

36. Campi R, Cotte J, Sessa F, et al. Robotic radical nephroureterectomy and segmental ureterectomy for upper tract urothelial carcinoma: a multi-institutional experience. World J Urol 2019;37:2303-11.

37. Dalpiaz O, Ehrlich G, Quehenberger F, et al. Distal ureterectomy is a safe surgical option in patients with urothelial carcinoma of the distal ureter. Urol Oncol 2014;32:34.e1-8.

38. Hoffman A, Yossepowitch O, Erlich Y, et al. Oncologic results of nephron sparing endoscopic approach for upper tract low grade transitional cell carcinoma in comparison to nephroureterectomy - a case control study. BMC Urol 2014;14:97.

39. Jang C, Lee CU, Sung SH, et al. Propensity-score matched comparison of oncologic and functional outcomes between radical nephroureterectomy and segmental ureterectomy Eur Urol Suppl 2019;18:e2164. 